\title{
Interactive Visual Tools for Spatial Multicriteria Decision Making
}

\author{
Gennady L. Andrienko and Natalia V. Andrienko \\ Fraunhofer AiS -Autonomous Intelligent Systems Institute \\ Schloss Birlinghoven, Sankt-Augustin, D-53754 Germany \\ WWW: http://www.ais.fhg.de/and/ \\ voice $+49-2241-142486$, fax $+49+2241-142072$ \\ gennady.andrienko@ais.fhg.de
}

\begin{abstract}
Spatial decision making is a complex cognitive process which requires appropriate support by interactive maps and other computer graphics. We develop tools to facilitate multicriteria evaluation of options by individuals as well as tools for analysis of results of voting in group decision making. Spatial distribution of options is represented by interactive map in combination with analysis of multidimensional attribute characteristics of decision options in statistical graphics.
\end{abstract}

\section{Categories and Subject Descriptors}

H.5.2 [Information Systems]: User Interfaces - Graphical user interfaces, Interaction styles.

\section{General Terms}

Design, Human Factors.

\section{Keywords}

Interactive tools, spatial decision support, multicriteria optimization.

\section{INTRODUCTION}

Our research work is mainly devoted to support of exploratory analysis of spatially referenced data. We investigate the potential of interactive dynamic maps [Andrienko and Andrienko 1999a] and their combination with methods of data mining [Andrienko and Andrienko 1999b]. In particular, we explore the opportunities of application of these tools to the task of spatial multicriteria decision making [Malczewski, 1999]. Our earlier experiences in this direction are described in [Andrienko, Jankowski and Andrienko, 2000; Jankowski, Andrienko and Andrienko, 2001]. In particular, we developed a series of map-centered displays and interactive procedures that facilitate individual decision making.

In this presentation we want to demonstrate some new developments not covered by the previous papers. The tools for decision making will be demonstrated on the example data set with characteristics of skiing resorts of the canton Wallis (Valais) in Switzerland. The attributes characterizing the resorts (such as

Permission to make digital or hard copies of all or part of this work for personal or classroom use is granted without fee provided that copies are not made or distributed for profit or commercial advantage and that copies bear this notice and the full citation on the first page. To copy otherwise, or republish, to post on servers or to redistribute to lists, requires prior specific permission and/or a fee.

Advanced Visual Interfaces '02, May 22-24, 2002, Trento, Italy.

Copyright 2002 ACM 1-58113-000-0/00/0000 ...\$5.00. maximum elevation, duration of skiing season, price for the skiing pass etc.) can be used as criteria in decision making, for example, in selection of a place to go on vacation.

Our system Descartes includes two groups of instruments: tools to be used by individual decision makers and tools for a facilitator of a process of collaborative decision making. These instruments will be briefly described in the next two sections.

\section{TOOLS TO SUPPORT INDIVIDUAL DECISION MAKING}

In constructing tools to support decision making one should take two essential points into account. First, two types of criteria need to be distinguished: benefit criteria (bigger values are better) and cost criteria (smaller values are better). Second, criteria may have different relative importance for the decision maker. Accordingly, all the instruments we propose allow the user to indicate the type of each criterion and to specify relative importance (weights) of criteria. Besides, the user may choose which of the attributes available she/he will use as criteria.

\section{1 "Utility signs"}

To support visual evaluation of spatially distributed options and search for suitable options on a map, we have developed a method for cartographic representation of characteristics of options called "utility signs". In our system we implemented two variants of utility signs: utility bar charts and utility wheels.
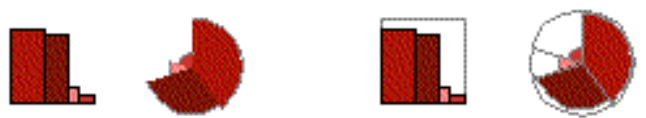

Figure 1. Utility bar charts and utility wheels.

A utility sign consists of several graphical elements (Figure 1): bars in utility bar charts and circle segments in utility wheels. Each element corresponds to one of the attributes under consideration (decision criterion). One dimension of an element (height in a bar and radius in a circle segment) represents the value of the attribute for the object this sign stands for. When the attribute is a benefit criterion, the size is proportional to the value, for a cost criterion the inverse proportion is kept. Hence, better values are always represented by bigger sizes. The other dimension (width in a bar and angle in a circle segment) represents the importance of the criterion. When the user interactively changes the weights of the criteria, the signs on the map are immediately redrawn. 
By the construction of a sign, the total area of all its elements shows approximately the "goodness", or utility, of the object this sign stands for. Hence, a decision maker needs to look on the map for signs with largest areas.

To simplify estimation of areas of utility signs and, thereby, visual evaluation of options, the signs can be supplemented with frames showing the maximum area (see Figure 1 on the right). This area corresponds to best possible values of all the attributes.

To facilitate visual analysis, we implemented 2 interactive features: visual search for options with a given quality (described in our previous publications), and visual comparison for utility bars for different options.

\subsection{Visual comparison technique for utility bars}

Earlier we have designed and implemented a "visual comparison" operation for a single numeric attribute represented on a map by painting or standalone bars [Andrienko and Andrienko 1999a]. We found this operation very useful for comparison of object characteristics and for exploration of value distribution. Recently we implemented a similar procedure to multidimensional data about decision options. Thus, Figure 2 demonstrates the values of three decision criteria for spatially distributed options are shown in comparison to values for a user-selected reference option (Torrent). Upward oriented bars represent values better than the reference option has, i.e. bigger values for benefit criteria and smaller values for cost criteria. Downward oriented bars represent worse values. The absence of a bar signifies the value equal to the corresponding value for the reference object. For example, one can see from the picture that "Crans Montana" is better evaluated than Torrent according to 2 criteria, and has a close value for the $3^{\text {rd }}$ criterion.

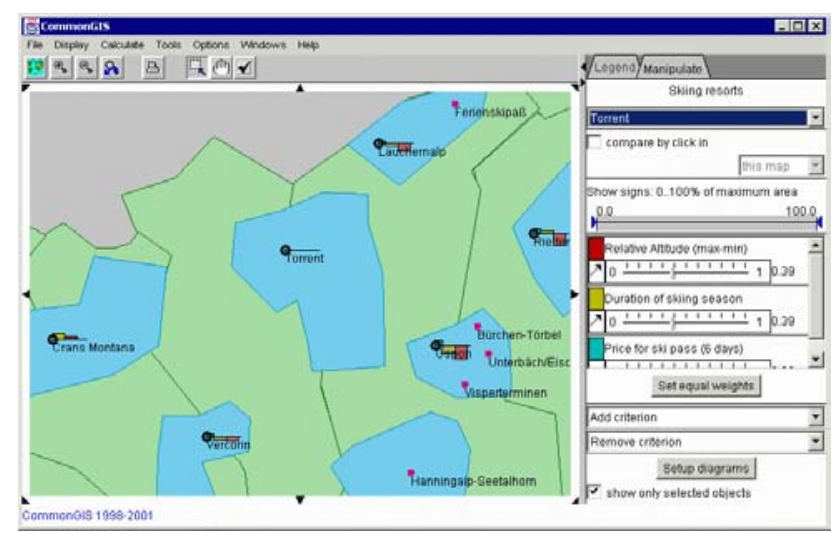

Figure 2. A map with comparison of the options to "Torrent".

The map in Figure 2 demonstrates an additional useful feature: it is possible to omit drawing of diagrams for objects the user is not interested in. When the check box "show only selected objects" is switched on, the system will only draw diagrams for objects explicitly selected by the user.

\subsection{Parallel coordinates plot and computation of integrated scores}

The visualization method known as parallel coordinates plot [Inselberg 1998] is applied when it is necessary to consider simultaneously multiple attributes (usually more than two).
Hence, it is potentially applicable in multicriteria decision making. However, the plot needs to be specifically constructed to account for peculiarities of decision making tasks: different directions of criteria (benefit or cost) and variation of weights.

In the variants of parallel coordinates plot we propose for decision making the axes for benefit and cost criteria have different orientation: left to right vs. right to left. The orientation is indicated by arrows. With such a solution, the best values of each attribute are always on the right, and the worst on the left. This makes it easy to estimate visually how good any specific option is: the closer to the right edge of the plot is a line, the better is the option.

Difference in relative weights of criteria can be reflected by variation of lengths of the axes: the more important is a criterion, the longer is the corresponding axis (see Figure 3). Due to this transformation lines of options surpassing others in more important criteria shift visually more to the right ("good") pole of the plot.

Visual estimation of goodness of options can be reinforced by application of existing computational methods for multi-criteria evaluation. Our system computes aggregated scores of options according to the so-called "Ideal point" method [Hwang and Yoon, 1981]. The method uses the weights assigned to the criteria by the decision maker. Results of computation are represented on the parallel coordinate plot together with the source data. Thus, in the plot shown in Figure 3 the computed scores are represented on the axis second from the bottom, and the bottom axis reflects ranking of the options with respect to the scores received. Such a display significantly helps in understanding and verification of the outcome of automatic evaluation. It also allows interactive analysis of sensitivity of the aggregated scores to changes of the weights. When the user alters any of the weights, the scores are immediately re-computed, and the results are reflected in the plot.

The plot may be linked to a map showing locations of the options (Figure 3). The map may represent the aggregated scores or the ranking of the options (for example, by degrees of darkness of painting) and change dynamically in parallel with changes in the plot.

\section{4 "Dynamic query"}

A useful tool for decision making is "dynamic query" [Ahlberg et al. 1992]. With this tool, which is available in our system, the user can interactively set and dynamically alter constraints on attribute values. In response to user's manipulations all the displays of the system are immediately redrawn to show only objects satisfying the constraints. A decision maker can impose constraints not only on source attributes but also on results of computation. Thus, applying "dynamic query" to the ranking of options according to the aggregated scores, the user can make all displays show only a selected number of top-scoring options (the map and the parallel coordinates plot in Figure 3 show five best options). When the decision maker alters the weights of the criteria, the system recomputes the scores and the ranking, applies the constraints to the new results obtained, and makes the map and other displays show the new set of best options (Figure 4). 


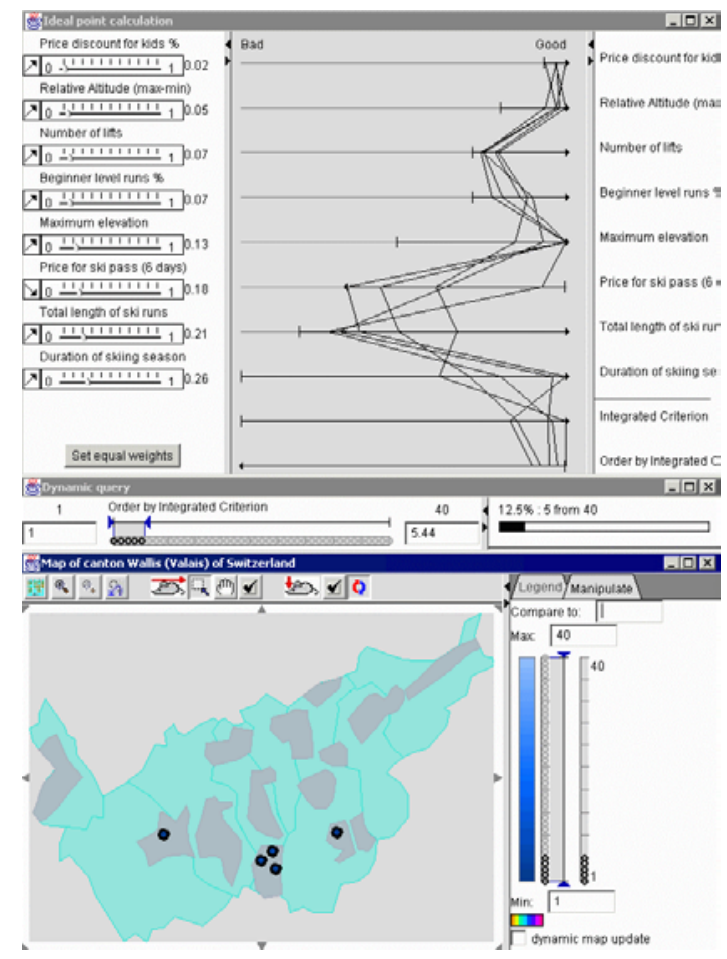

Figure 3. Five best options are selected using "dynamic query".

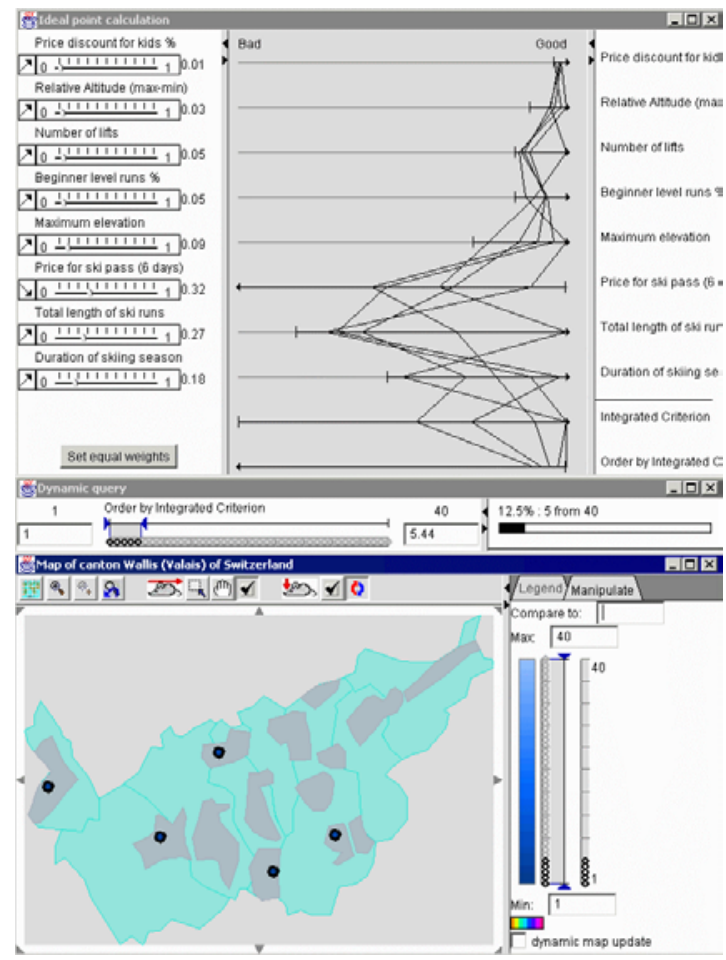

Figure 4. The set of five best options has changed (in comparison to that in Figure 3) after the user modified the weights of some criteria.

\subsection{Sensitivity analysis and visualization of results}

Besides finding the best options among available, it is important to understand how sensitive the decision procedure is to slight changes of the weights of the criteria. One possibility is to "shake" the weights manually, by moving corresponding sliders around their current positions. This procedure can be automated. We implemented a sensitivity analysis method in the system, which iteratively changes weights of selected criteria in userspecified intervals. For each criterion the user may select the desired number of iterations (Figure 5).

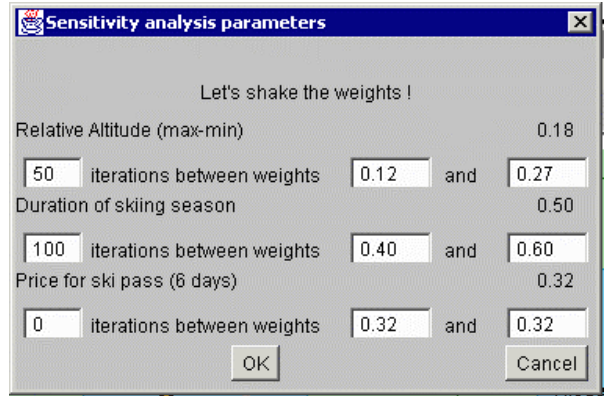

Figure 5. A panel for specification of the parameters for the sensitivity analysis. The user has selected 50 iterations for the first criterion and $\mathbf{1 0 0}$ iterations for the second.

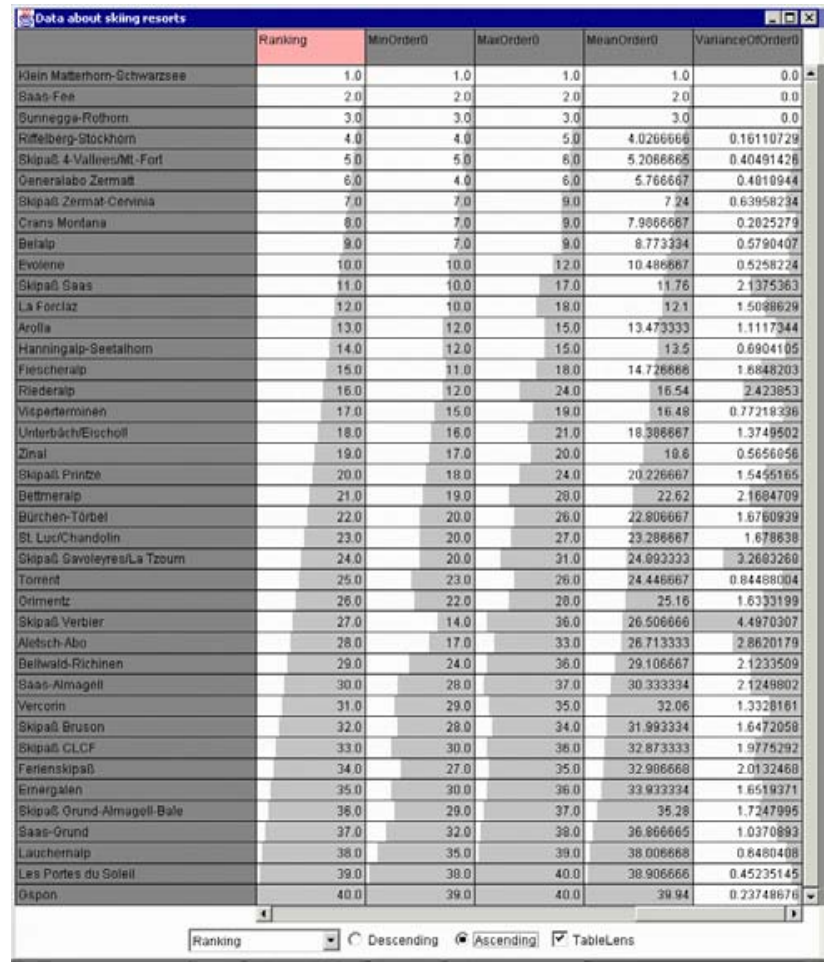

Figure 6. Combined symbolic and graphical representation of sensitivity analysis results. The first column corresponds to the current ranking, the next 3 columns show minimal, maximal, and mean rankings over 150 iterations. The last column shows the variance of ranks. 
The results are added to the table with the source data and can be graphically represented on the map or other displays. For example, bar charts can show the current, minimal, mean, and maximal ranking of each object.

Another interesting possibility is to use the "table lens" technique [Rao and card, 1994] (see Figure 6). After sorting the table according to the current ranks, it is clearly visible that the mean ranks are strongly following them. The curves for the minimal and maximal ranks are also similar to that for the current ranks but have some slight deviations. Very interesting is the curve for the variance. One can see that for the 3 best options the variance is 0 , i.e. they remained the best over all iterations. For the next 7 options the variance is very low, and variation of the ranks (the difference between maximal and minimal ranking) is smaller than 3. Finally, the biggest variance (and, respectively, the most sensitive result) has the $27^{\text {th }}$ option which balanced between $14^{\text {th }}$ and $38^{\text {th }}$ ranks. However, this option is, apparently, not interesting from the perspective of decision making.

\section{TOOLS TO SUPPORT GROUP DECISION MAKING}

One of possible scenarios of group decision making supposes that the group members individually analyze the space of options and then vote about the options. Descartes includes tools that allow the person leading the process of group decision making (facilitator) to analyze the results of voting.

In order to get material for application of the tools, we asked our colleagues to provide their votes about the skiing resorts in the form of assigning them to one of the three classes "preferred", "agreeable", and "rejected". Some persons evaluated only a subset of the options, and we had to deal with one more class, "non-considered". The vote of each person was stored as a column of a table with rows corresponding to the options.

The following tools provided by Descartes could be useful for a facilitator of a group decision making process:

1) Automatically count for each option the number of people having assigned it to each of the classes (or, in other words, count for each row of the table the number of occurrences of different values in a specified subset of columns). The counts thus obtained can be visualized using all kinds of displays available in the system.

2) "Dynamic query" allows the facilitator to choose a reasonable subset of options for further consideration (options with the largest number of supporters and the smallest number of opponents).

3) With a parallel coordinates plot the facilitator can view and compare individual votes.

4) Automated calculation of average values and variances of values in the specified subset of columns helps to analyze the degree of agreement between group members about each option.

\section{IMPLEMENTATION NOTES}

All mentioned tools are implemented in Java (JDK 1.1) and are available in the Descartes system. The system can run both locally on any computer with the Java runtime environment installed or in the Internet within standard WWW browsers.

\section{ACKNOWLEDGMENTS}

We thank Dr. Hans Voss (Fraunhofer AiS) for useful comments and fruitful discussion about our work. Prof. Piotr Jankowski from the University of Idaho inspired our interest to spatial decision support. We are grateful to Mr. Andrey Martinkin for implementation of table lens technique in Descartes, Ms. Sabine Mull and Mr. Felix Kollbach for collecting and compiling the data about skiing resorts in Wallis. The work was partly funded by $\mathrm{BMBF}$ in project KogiPlan (reference number VFG0003B, 1.10.2000-31.3.2003).

\section{REFERENCES}

[1] Ahlberg, C., Williamson, C., and Shneiderman, B. "Dynamic queries for information exploration: an implementation and evaluation". In Proceedings ACM CHI'92 (ACM Press), 1992, 619-626

[2] G. Andrienko and N. Andrienko, "Interactive Maps for Visual Data Exploration", International Journal of Geographical Information Science, 13 (4), 1999, pp.355374.

[3] Andrienko, G. and Andrienko, N. "Data Mining with C4.5 and Cartographic Visualization". In N.W.Paton and T.Griffiths (eds.) User Interfaces to Data Intensive Systems 1999. IEEE Computer Society, Los Alamitos, CA, pp. 162165

[4] Andrienko, G., Jankowski, P., Andrienko, N. "Data mining approach to reducing complexity of multiple criteria spatial decision problem" In P.Forer, A.G.O.Yeh, J.He (eds.) Proceedings of 9th International Symposium on Spatial Data Handling, Beijing, China, 10-12 August 2000. International Geographical Union, 2000, pp.3a15-3a28

[5] Inselberg, A. Visual Data Mining with Parallel Coordinates, Computational Statistics, 13 (1), 1998, pp.47-63

[6] Jankowski, P., Andrienko, N., and Andrienko, G. "MapCentered Exploratory Approach to Multiple Criteria Spatial Decision Making", International Journal Geographical Information Science, 15 (2), 2001, pp.101-127

[7] P.Jankowski, A.Lotov, and D.Gusev. Multiple Criteria Trade-off Approach to Spatial Decision Making. In J.-C. Thill (Ed.) Spatial Multicriteria Decision Making and Analysis: A Geographical Information Sciences Approach, Ashgate, Brookfield, VT, 1999

[8] Hwang, C,L., Yoon, K. (1981). Multiple Attribute Decision Making: Methods and Applications. Berlin, Springer-Verlag.

[9] Malczewski, J. 1999. GIS and Multicriteria Decision Analysis. New York: John Wiley \& Sons

[10] Rao,R., and Card,S.K. (1994). The Table Lens: Merging Graphical and Symbolic Representations in an Interactive Focus+Context Visualization for Tabular Information. Proceedings of CHI'94, Boston MA, ACM Press, pp.318$322,481-482$. 